\title{
National Institutes of Health Consensus Development Conference Statement: Management of Hepatitis B
}

Michael F. Sorrell, MD; Edward A. Belongia, MD; Jose Costa, MD; Ilana F. Gareen, PhD; Jean L. Grem, MD; John M. Inadomi, MD; Earl R. Kern, PhD; James A. McHugh, MD; Gloria M. Petersen, PhD; Michael F. Rein, MD; Doris B. Strader, MD; and Hartwell T. Trotter, MS*

$\mathrm{N}$ ational Institutes of Health (NIH) consensus and stateof-the-science statements are prepared by independent panels of health professionals and public representatives on the basis of 1) the results of a systematic literature review prepared under contract with the Agency for Healthcare Research and Quality (AHRQ); 2) presentations by investigators working in areas relevant to the conference questions during a 2-day public session; 3) questions and statements from conference attendees during open discussion periods that are part of the public session; and 4) closed deliberations by the panel during the remainder of the second day and morning of the third. This statement is an independent report of the panel and is not a policy statement of the National Institutes of Health or the U.S. government. The statement reflects the panel's assessment of medical knowledge available at the time the statement was written. Thus, it provides a "snapshot in time" of the state of knowledge on the conference topic. When reading the statement, keep in mind that new knowledge is inevitably accumulating through medical research.

Hepatitis B is a major cause of liver disease worldwide, ranking as a substantial cause of cirrhosis and hepatocellular carcinoma. The development and use of a vaccine for hepatitis B virus (HBV) has resulted in a substantial de-

See also:

Print

Related article. 111

Web-Only

Appendix

Conversion of graphics into slides cline in the number of new cases of acute hepatitis B among children, adolescents, and adults in the United States. However, this success has not yet been duplicated worldwide, and both acute and chronic HBV infection continue to represent important global health problems.

Seven treatments are currently approved for adult patients with chronic HBV infection in the United States: interferon- $\alpha$, pegylated interferon- $\alpha$, lamivudine, adefovir dipivoxil, entecavir, telbivudine, and tenofovir disoproxil fumarate. Interferon- $\alpha$ and lamivudine have been approved for children with HBV infection. Although available randomized, controlled trials (RCTs) show encouraging short-term results-demonstrating the favorable effect of these agents on such intermediate markers of disease as HBV DNA level, liver enzyme tests, and liver histologylimited rigorous evidence exists demonstrating the effect of these therapies on important long-term clinical outcomes, such as the development of hepatocellular carcinoma or a reduction in deaths. Questions therefore remain about which groups of patients benefit from therapy and at which point in the course of disease this therapy should be initiated.

We were charged with answering the following critical questions about the management of hepatitis $\mathrm{B}$ :

What is the current burden of hepatitis $B$ ?

What is the natural history of hepatitis B?

What are the benefits and risks of the current therapeutic options for hepatitis B?

Which persons with hepatitis B should be treated?

What measures are appropriate to monitor therapy and assess outcomes?

What are the greatest needs and opportunities for future research on hepatitis B?

At the conference, invited experts presented information relevant to these questions and a systematic literature review, prepared under contract with the AHRQ, was

* Panel statement from an NIH Consensus Development Conference held on 20-22 October 2008 at the National Institutes of Health, Bethesda, Maryland. For a list of the members of the NIH Consensus Development Panel and other participants, see the Appendix (available at www.annals.org).

Individuals who wish to cite this recommendation statement should use the following format: Sorrell MF, Belongia EA, Costa J, Gareen IF, Grem JL, Inadomi JM, et al. National Institutes of Health Consensus Development Conference Statement: management of hepatitis B. Ann Intern Med. 2009;150:104-10.

This article was published at www.annals.org on 6 January 2009. 
summarized. The evidence report, available at www.ahrq .gov/clinic/tp/hepbtp.htm, emphasizes RCTs with health outcomes as their end points. (A concise summary of the evidence report is available in this issue as a companion to this statement [1].) Conference attendees also provided oral and written comments in response to the conference questions, and we considered all of this evidence when preparing the consensus statement.

\section{What Is the Current Burden of Hepatitis B?}

An estimated 400 million people worldwide are living with chronic HBV infection. Each year, an estimated 500000 people die of cirrhosis and hepatocellular carcinoma caused by chronic infection and an additional 40000 people die of acute hepatitis B. The prevalence of HBV infection is uneven throughout the world, with significant burdens in Asia and the Pacific Islands, subSaharan Africa, the Amazon Basin, and Eastern Europe.

The incidence (rate of new cases) of acute HBV infection has decreased dramatically in the United States since the mid-1980s. This reduction can be attributed to the availability of an effective vaccine and widespread immunization of infants and high-risk populations. However, the number of people who have chronic HBV infection remains high because of the long duration of infection and influx of immigrants who have chronic infection. It is estimated that more than 1 million U.S. residents have chronic infection, which contributes to an estimated 2000 to 4000 deaths each year. National surveys indicate that $0.3 \%$ to $0.5 \%$ of U.S. residents have chronic infection, and $47 \%$ to $70 \%$ of these persons were born outside the United States. The prevalence of HBV infection is higher among people who were born in countries with a high HBV prevalence and members of subpopulations that have behavioral risk factors for HBV transmission, including injection-drug users and men who have sex with men. More comprehensive screening for $\mathrm{HBV}$ is needed for public health evaluation and management of chronically infected persons and their contacts.

The public health burden of HBV is almost entirely due to its long-term effects on liver function. Chronic HBV infection is a major cause of cirrhosis and hepatocellular carcinoma. In addition to the human suffering that these diseases cause, the social and economic costs are large. More than $\$ 1$ billion is spent each year for hepatitis B-related hospitalizations. The indirect costs of chronic HBV infection are harder to measure, but include reduced physical and emotional quality of life, reduced economic productivity, long-term disability, and premature death.

\section{What Is the Natural History of Hepatitis B? Acute Hepatitis B Infection}

Hepatitis B virus is transmitted through infected blood or body fluids that enter the body through mucous membranes, wounds, or injection (for example, by sharing needles or syringes). The virus also can be transmitted by sexual contact with an infected person or by perinatal exposure to an infected mother. Acute HBV infection can be symptomatic or asymptomatic. Symptomatic infection is rare in newborns and young children, but symptoms of acute hepatitis B occur more frequently in susceptible adults after infection. Hepatitis B surface antigen (HBsAg) is detectable in the blood within 4 to 10 weeks after infection. The typical incubation period is about 3 months, but it may be as long as 6 months before symptoms develop.

In adults, most acute HBV infections are self-limited, and patients recover completely after developing specific antibodies (anti-HBs) and clearing HBsAg from the blood. A small proportion of patients develop severe acute hepatitis B. The risk for severe acute hepatitis B may be increased in persons who are co-infected with hepatitis C virus $(\mathrm{HCV})$ or hepatitis $\mathrm{D}$ virus.

\section{Chronic Hepatitis B Infection}

A small proportion $(<5 \%)$ of adults develop chronic HBV infection with ongoing viral replication in the liver. Chronic infection occurs in almost all children who are infected with hepatitis B during the perinatal period and in up to $50 \%$ of children who become infected between 1 and 5 years of age. Most people who have chronic HBV infection in the United States have been infected since birth or early childhood, and these infections were probably acquired in countries where the prevalence of $\mathrm{HBV}$ infection is higher.

Chronic HBV infection has 3 major phases: immune tolerant, immune active, and inactive carrier phases (Figure). Patients usually pass from one phase to the next, but some who transition from the immune-active phase to the inactive carrier phase subsequently experience reactivation and redevelop chronic hepatitis (immune active phase). The immune-tolerant phase is characterized by active viral replication in the liver but little or no evidence of disease activity. This phase occurs in almost all children who are infected at birth and is characterized by high levels of HBV DNA in the blood without liver inflammation. In this phase, liver biopsy is normal or shows only minimal inflammation. The immune-tolerant phase may last for decades in children who are infected during the perinatal period. Liver disease does not seem to progress during this phase.

Most children and adults will eventually progress from the immune-tolerant phase to the immune-active phase. In this phase, the immune response to HBV becomes more robust, with evidence of liver inflammation and elevated levels of liver enzymes in the blood. A liver biopsy will show inflammation with or without fibrosis (scarring). In both the immune-tolerant and immune-active phases, persons usually have detectable levels of hepatitis $\mathrm{B}$ e antigen ( $\mathrm{HBeAg})$. Some patients who are infected with HBV have no detectable HBeAg in any stage; such patients may have

20 January 2009 $\mid$ Annals of Internal Medicine $\mid$ Volume 150 • Number 2|105 


\section{Figure. Natural history of chronic hepatitis B virus infection.}

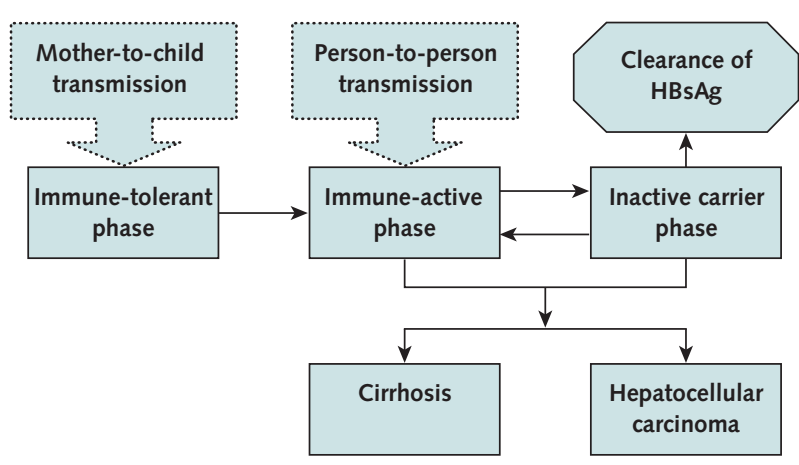

$\mathrm{HBsAg}=$ hepatitis B surface antigen.

a different natural history. The presence of $\mathrm{HBeAg}$ generally indicates high levels of HBV DNA in the blood. Elevated levels of HBV DNA in the blood are associated with liver inflammation in the immune-active phase.

Most people who have chronic HBV infection will eventually enter the inactive carrier phase as they clear $\mathrm{HBeAg}$ and develop anti-HBe (HBeAg seroconversion). Seroconversion of $\mathrm{HBeAg}$ usually marks the transition from the immune-active phase to the inactive carrier phase and is accompanied by undetectable or low levels of HBV DNA, which leads to normalization of levels of alanine aminotransferase (ALT), and reduced liver inflammation. Hepatitis B virus DNA is still present in the blood during the inactive carrier phase, but at lower levels than during the immune-active phase. Persons in the inactive carrier phase have a low risk for hepatocellular carcinoma, and liver abnormalities generally do not progress to more severe disease. Persons who become HBsAg-negative usually develop antibodies (anti-HBs) and can be considered to have resolved hepatitis $\mathrm{B}$. A small proportion of these persons is found to have detectable HBV DNA in serum, although the levels are low and observed only intermittently. This state has been referred to as "occult" or "latent" hepatitis B. The natural history of this condition is not well known but it is unlikely to be associated with progressive liver disease. Most persons who have resolved hepatitis B have detectable HBV DNA levels in the liver, and the disease may be reactivated by severe immunosuppression.

The long-term course of chronic HBV infection varies substantially. Active liver disease (immune-active phase) may convert to inactive disease but then reactivate, with the reappearance of high levels of HBV DNA. During active disease periods, progression to advanced fibrosis occurs at a variable rate. Disease progression also varies on the basis of the age at which primary infection occurred. Persons who are infected as adults or adolescents generally become inactive carriers after they clear $\mathrm{HBeAg}$. In contrast, persons who were infected at birth or in early childhood have a prolonged immune-tolerant phase, and evi- dence shows that the disease continues to progress even after $\mathrm{HBeAg}$ disappears in some of these patients. Lifelong monitoring is indicated.

\section{Cirrhosis and Hepatocellular Carcinoma}

Multiple studies in diverse populations have reported that chronic HBV infection is a strong risk factor for hepatocellular carcinoma. Adults with chronic HBV infection that was acquired in the perinatal period develop hepatocellular carcinoma at a rate of about 5\% per decade, which is approximately 100 -fold higher than the rate among uninfected persons. Hepatocellular carcinoma is most common in developing countries where hepatitis B is endemic. Hepatocellular carcinoma is rare in the United States, although the incidence has increased over the past 20 years. An unknown but substantial proportion of cases, however, can be attributed to HCV. The mortality rate for hepatocellular carcinoma is extremely high, except in selected patients who undergo liver resection or transplantation.

People who remain in the immune-active phase of HBV infection for a long time have the highest risk for cirrhosis and hepatocellular carcinoma. The risk for cirrhosis and hepatocellular carcinoma remains low for persons in the immune-tolerant phase and the inactive carrier phase. Important predictors of cirrhosis and hepatocellular carcinoma include prolonged elevation of HBV DNA in the blood, elevated ALT level, and presence of HBeAg. Patients who have chronic HBV infection may rarely develop hepatocellular carcinoma in the absence of cirrhosis; this generally occurs in younger patients. Characteristics of both the virus and the infected person may influence the likelihood of developing cirrhosis or hepatocellular carcinoma. Long-term follow-up studies have shown that HBV genotype $\mathrm{C}$ infection poses an increased risk for cirrhosis and hepatocellular carcinoma; this genotype circulates mainly in Asia and the Pacific Islands. Other risk factors for hepatocellular carcinoma in people with chronic HBV infection include male sex, older age, and family history of hepatocellular carcinoma. Co-infection with HCV increases the risk for cirrhosis and hepatocellular carcinoma.

The mechanisms through which HBV causes hepatic injury and triggers hepatocellular carcinoma are not well understood. The roles of host genetic factors and variation in host immune response are also not known, which indicates a need for future research.

\section{What Are the Benefits and Risks of the Current Therapeutic Options for Hepatitis B?}

Currently, 7 agents have been approved by the U.S. Food and Drug Administration for use in the treatment of adults with HBV. These agents, categorized as either interferons (interferon- $\alpha 2 \mathrm{~b}$ and peginterferon- $\alpha 2 \mathrm{a}$ ) or nucleoside or nucleotide analogues (lamivudine, adefovir, entecavir, tenofovir, and telbivudine), may be used as monotherapy or in combination. Interferon use has a defined, self-limited course; in contrast, therapy with nucle- 
oside or nucleotide analogues can be long-term, often indefinite, treatment.

The major goals of anti-HBV therapy are to prevent the development of progressive liver disease, specifically cirrhosis and liver failure, and prevent the development of hepatocellular carcinoma and subsequent death. To date, no conclusive evidence from RCTs of anti-HBV therapy has demonstrated a beneficial impact on any of these primary clinical outcomes because cirrhosis, hepatocellular carcinoma, and death often do not occur for many years after infection with HBV and would therefore require long-term evaluation of therapy to demonstrate benefit. As a consequence, most published reports of anti-HBV therapy use changes in short-term virologic, biochemical, and histologic parameters to infer the likelihood of long-term benefit. It is important to understand the limitations of this practice when assessing potential benefit.

The NIH Biomarkers Working Group has defined a clinical end point as "a characteristic or variable that reflects how a patient feels or functions, or how long a patient survives" and a surrogate end point as "a biomarker intended to substitute for a clinical end point." A surrogate end point is expected to predict clinical benefit, harm, or lack of benefit or harm. The effect of the proposed therapy on the surrogate marker must predict the effect on the clinical outcome and must be part of the causal pathway. In studies of hepatitis B therapy, loss of HBsAg, HBV DNA level, $\mathrm{HBeAg}$ or antibody status, ALT level normalization, and improvement in liver histology have been advanced as surrogate end points. Review of the natural history of HBV suggests that the loss of HBsAg may be the best surrogate because it indicates immunity to HBV, decreased risk for development of cirrhosis and hepatocellular carcinoma, and improved survival. Unfortunately, such seroconversion rarely occurs in response to therapy. Several studies have identified elevated HBV DNA level as a predictor of development of cirrhosis and hepatocellular carcinoma. Suppression of HBV DNA has been associated with improvement of ALT and improved histology. What is less clear is whether treatment-induced decreases in HBV DNA levels are associated with improved clinical outcomes. Thus, changes in these proposed surrogates may not predict improved clinical outcomes. In the absence of long-term RCTs with clinical outcomes, the use of intermediate biomarkers may be the next best option.

The benefits of therapy for hepatitis B must be viewed in the proper context. Approved therapy is associated with improvements in certain intermediate biomarkers, with low-quality evidence showing a correlation with clinical outcome. All approved treatments decrease HBV DNA levels. The extent of the decline is greater and the time to decline shorter with the use of nucleoside or nucleotide analogues compared with interferon. Likewise, all approved therapies have been associated with some degree of $\mathrm{HBeAg}$ loss or seroconversion, decreases in ALT level, and improvement in liver histology.
Each category of treatment, interferons or nucleoside or nucleotide analogues, has unique advantages and risks associated with administration of the drug. An advantage of interferon is that it is given for a defined course (16 to 48 weeks) and is not associated with the development of antiviral resistance. The use of interferon requires subcutaneous injection and is associated with systemic side effects, such as headache, nausea, flu-like symptoms, depression, and some hematologic abnormalities. Nucleoside and nucleotide analogues are administered orally, are associated with more profound HBV DNA suppression than interferon, and may be safely used in previous nonresponders to interferon therapy. However, if prematurely discontinued, these drugs are associated with resurgence of HBV DNA levels or reactivation of hepatitis. In addition, long-term use of these drugs is compromised by the development of resistance. Several of the nucleoside and nucleotide analogues are associated with renal toxicity, myopathy (muscle weakness or pain), and mitochondrial toxicity.

The evidence available at this time does not permit concrete recommendations regarding selection of a particular therapeutic course. Health care providers should discuss the risks and benefits of treatment options with patients to arrive at the best possible decisions.

\section{Which Persons With Hepatitis B Should Be TREATED?}

From the time of initial diagnosis, optimal management of HBV infection requires a lifetime of routine monitoring, even when patients are asymptomatic. We wish to emphasize that provider and patient education are key to ensuring ongoing adherence with routine disease and treatment response monitoring and with therapy.

\section{Patients for Whom Therapy Is Indicated}

Therapy is indicated for patients with rapid deterioration of liver function and patients with decompensated cirrhosis, defined as cirrhosis with such complications as ascites, hepatic encephalopathy, or hemorrhage due to portal hypertension. No RCTs have been conducted in these patient populations. However, clinical experience supports a reduction in adverse clinical outcomes through antiviral therapy with nucleosides or nucleotides. Interferon- $\alpha$ and pegylated interferon- $\alpha$ therapies are contraindicated in this group because of the risk for hepatic failure.

Patients who have compensated cirrhosis are at an increased risk for clinically important complications. A single placebo-controlled RCT demonstrated a clinically relevant improvement in the stage of cirrhosis (Child-TurcottePugh score) and a borderline-significant reduction in the incidence of hepatocellular carcinoma with therapy. This study was halted for benefit by the data safety monitoring board on the basis of a specified interim analysis that demonstrated an improvement in the Child-Turcotte-Pugh score in those receiving active anti-HBV therapy. Therefore, we agree that therapy is indicated for these patients.

20 January 2009|Annals of Internal Medicine $\mid$ Volume 150 • Number 2|107 
Observational studies indicate that patients with HBV who receive immunosuppressive or cancer chemotherapy for other medical conditions are at high risk for developing exacerbation of hepatitis, including those who have chronic $\mathrm{HBV}$ and those who are in the inactive $\mathrm{HBsAg}$ carrier phase. In these patients, it is important to start antiviral therapy for hepatitis B before initiating immunosuppressive therapy. Antiviral therapy should be maintained throughout the course of treatment.

Women who are HBsAg-positive have a very high risk for vertical transmission of $\mathrm{HBV}$ to their infants. Therefore, it is currently recommended that infants of $\mathrm{HBsAg}$ positive women receive hepatitis $\mathrm{B}$ immunoglobulin and hepatitis $B$ vaccination within 12 hours of birth; this has been demonstrated to substantially reduce the risk for perinatal transmission. It is important that these infants receive a complete set of 3 vaccinations and long-term follow-up.

\section{Patients for Whom Therapy May Be Indicated}

Most trials of anti-HBV therapies conducted for drug approval purposes have enrolled patients who have chronic HBV with high HBV DNA levels and signs of liver inflammation as reflected by elevated ALT levels or histology. The decision to treat is affected by knowledge about the natural history of these patients in the absence of therapy. An elevated ALT level indicates active liver inflammation and is considered a predictor of likelihood of disease progression.

Patients in the immune active phase (sometimes referred to as immune clearance) may be treatment candidates after consideration of various prognostic factors. The immune active phase is defined by the presence of elevated HBV DNA levels, with or without $\mathrm{HBeAg}$, and evidence of active inflammation (ALT level elevation or active inflammation on liver histology). The available RCTs provide evidence that selected patients treated with anti-HBV therapy have decreases in HBV DNA levels and improvement in ALT levels. The onset of complications from chronic HBV generally increases in patients around age 40 years. Younger HBeAg-positive patients may undergo spontaneous HBeAg seroconversion; therefore, it is reasonable to monitor this group without therapy unless evidence of progressive liver disease is found. If spontaneous seroconversion does not occur by the late 30 s or early 40 s and active inflammation is present, as reflected by ALT level elevation or inflammation or fibrosis on liver biopsy, therapy may be indicated.

Patients in the reactivation phase of chronic HBV infection, defined as having elevated HBV DNA levels and evidence of liver inflammation, usually should be treated. In general, these patients have evidence of liver inflammation in association with lower HBV DNA levels compared with the HBeAg-positive patients. Therefore, a lower threshold of HBV DNA levels in the presence of liver inflammation might justify therapy.
Several prognostic factors for disease progression may be considered in the decision to treat, including male sex, genotype (genotype C), a family history of hepatocellular carcinoma, and ongoing alcohol abuse. Co-infection with HIV, HCV, or hepatitis D virus increases the risk for adverse clinical outcomes. If the HIV infection requires treatment, then hepatitis B also should be treated. Combination therapy with nucleoside or nucleotide analogues is required to avoid the emergence of resistance and to provide optimal reduction in the replication of both viruses. The antiviral therapies must be selected in view of the potential for cross-resistance. If HIV is not treated, then the decision to treat the HBV infection with therapy that targets HBV replication should follow guidelines for HBV monoinfection, except a lower threshold for HBV DNA might trigger treatment. Some information suggests that a normal ALT level in co-infected patients does not exclude the presence of active liver inflammation.

\section{Patients for Whom Immediate Therapy Is Not Routinely Indicated}

Certain patients have a lower risk for adverse clinical outcomes. These patients may be identified through various clinical features (such as younger age) and absence of indicators of hepatic inflammation. As such, we suggest that younger patients in the immune-tolerant phase, those in the inactive carrier phase, and those who have latent $\mathrm{HBV}$ infection do not meet the criteria for therapy.

Therapy is not recommended for patients who are in the immune-tolerant phase, which includes the presence of HBsAg, high HBV DNA levels, normal ALT levels, and liver histology with mild or minimal inflammation and fibrosis. Typically, such patients have not been included in prospective RCTs. As mentioned, retrospective data suggest that some patients who have baseline ALT levels in the normal range may, rarely, have adverse outcomes. Also, ALT values can vary over time. Serial monitoring of ALT levels may help identify those persons who have ALT levels that are persistently in the normal range and who thus have a favorable prognosis. Careful surveillance is reasonable for such patients.

Therapy is also not recommended for patients who are in the inactive carrier or low replicative phase, defined by the presence of HBsAg, low HBV DNA levels, normal ALT levels, and liver histology with mild or minimal inflammation and fibrosis. The presence of latent HBV infection, defined as detection of HBV DNA in the absence of $\mathrm{HBsAg}$, is not an indication for therapy. The natural history of this condition is not known, nor are the response to or outcomes of therapy.

Additional factors related to the patient must be considered when therapy is being contemplated. Treatment may not be indicated in patients for whom concurrent serious medical conditions preclude expectation of improved outcomes with therapy. This is because the risk for 
death from the coexisting medical condition is high and complications from HBV-associated liver disease are therefore unlikely to contribute to morbidity and mortality. AntiHBV therapy will be most effective in those patients who follow the prescribed regimen for therapy. Thus, patients who are nonadherent to a prescribed anti-HBV regimen are unlikely to benefit from therapy.

If a decision is made not to institute anti-HBV therapy as discussed, it is important to continue monitoring ALT values at regular intervals. If the ALT level becomes elevated, the patient should be referred to a liver specialist for consideration of therapy.

\section{What Measures Are Appropriate to Monitor Therapy AND Assess OUtCOMES?}

The goal of anti-HBV therapy is to prevent progression of liver disease. During the course of therapy, treatment response may be monitored by using biochemical, virologic, serologic, and histologic indices. The preferred measure of virologic activity is quantitation of HBV DNA with an assay, such as reverse transcriptase-polymerase chain reaction, that provides a wide dynamic range. Hepatitis $\mathrm{B}$ surface antigen loss and seroconversion are associated with durable suppression of HBV DNA; however, this is uncommonly achieved in the short term with current therapy.

Although various monitoring practices have been recommended, no clear evidence exists for an optimal approach. One proposed management algorithm used during therapy involves measuring HBV DNA and ALT levels every 12 weeks and $\mathrm{HBeAg}$ or anti-HBe levels every 24 weeks in patients who are HBeAg-positive. Sex-specific differences in the upper limits of normal for ALT levels deserve consideration when this test is used to monitor therapeutic response. For patients who are HBeAg-positive and achieve a complete response (undetectable HBV DNA), seroconversion to anti-HBe may offer the opportunity to discontinue therapy after 6 to 12 months of "consolidation." During this time, periodic monitoring of $\mathrm{HBV}$ DNA and HBeAg status should continue because relapse remains a possibility. Therapy should be continued in patients with cirrhosis. These practices are based on limited data and represent an opportunity for continued research. We support the adoption of standardized monitoring practices during clinical trials.

The balance of benefits and harms associated with screening for hepatocellular carcinoma is unknown and is an area for future research.

\section{What Are the Greatest Needs and OpPortunities for Future Research on Hepatitis B? \\ General}

The long duration of illness and the complex course of $\mathrm{HBV}$ infection create major challenges for effective basic and clinical research. Multicenter clinical trials need to incorporate extended follow-up, measuring health outcomes in specific populations that are known to have high rates of infection. Even in the setting of approved drugs, RCTs, including placebo-controlled studies, are still indicated. The chronic course of hepatitis B has encouraged acceptance of intermediate indicators of therapeutic efficacy on the basis of observational studies, a process that may lead to biased estimates of therapeutic effect.

To ensure that the results of different studies are comparable or may be combined for analysis, such studies should be conducted by using standardized protocols, including definitions of populations; regimens; clinical definitions; diagnostic methods; intervals and techniques for follow-up; and, most important, standard definitions of improvement. Studies involving multiple interventions, end points, populations, and comparisons must account statistically for this structure. Attention must be paid to the connections or disconnections between statistical significance and clinical and heuristic consequence.

\section{Research Priorities}

1. Representative, prospective cohort studies to define the natural history of the disease.

2. Large, multicenter RCTs, including placebo-controlled trials, of monotherapy and combined therapies with measurement of the effect of treatment on clinical health outcomes.

3. Role of HBV replication in host response and carcinogenesis.

4. Risks and benefits of antiviral therapy and other strategies to reduce vertical transmission in pregnancy.

5. The quantitative and qualitative characteristics of the immune response in different phases of $\mathrm{HBV}$ infection.

6. The risks and benefits of screening for hepatocellular carcinoma in chronic hepatitis B.

\section{CONCLUSION}

The most important predictors of cirrhosis or hepatocellular carcinoma in persons who have chronic HBV are persistently elevated HBV DNA and ALT levels in blood. Other risk factors include $\mathrm{HBV}$ genotype $\mathrm{C}$ infection, male sex, older age, family history of hepatocellular carcinoma, and co-infection with HCV or HIV.

The major goals of anti-HBV therapy are to prevent the development of progressive disease, specifically cirrhosis and liver failure, as well as hepatocellular carcinoma development and subsequent death. To date, no RCTs of anti-HBV therapies have demonstrated a beneficial impact on overall mortality, liver-specific mortality, or development of hepatocellular carcinoma.

Most published reports of hepatitis therapy use changes in short-term virologic, biochemical, and histologic parameters to infer likelihood of long-term benefit. Approved therapies are associated with improvements in intermediate biomarkers, including $\mathrm{HBV}$ DNA, $\mathrm{HBeAg}$ 


\section{Table. Criteria Useful in Determining for Whom Therapy Is Indicated}

Patients for whom therapy is indicated

Patients who have acute liver failure, cirrhosis and clinical complications, cirrhosis or advanced fibrosis and HBV DNA in serum, or reactivation of chronic HBV after chemotherapy or immunosuppression

Infants born to women who are HBsAg-positive (immunoglobulin and vaccination)

Patients for whom therapy may be indicated

Patients in the immune-active phase who do not have advanced fibrosis or cirrhosis

Patients for whom immediate therapy is not routinely indicated

Patients with chronic hepatitis B in the immune-tolerant phase (with high levels of serum HBV DNA but normal serum ALT levels or little activity on liver biopsy)

Patients in the inactive carrier or low replicative phase (with low levels of or no detectable HBV DNA in serum and normal serum ALT levels)

Patients who have latent HBV infection (HBV DNA without HBsAg)

$\mathrm{ALT}=$ alanine aminotransferase; $\mathrm{HBsAg}=$ hepatitis $\mathrm{B}$ surface antigen; $\mathrm{HBV}=$ hepatitis B virus.

loss or seroconversion, decreases in ALT levels, and improvement in liver histology (Table).

Although various monitoring practices have been recommended, no clear evidence exists for an optimal approach.

The most important research needs include representative prospective cohort studies to define the natural history of the disease and large RCTs of monotherapy and combined therapies, including placebo-controlled trials, that measure the effects on clinical health outcomes.

We recommend routine screening for hepatitis $\mathrm{B}$ of newly arrived immigrants to the United States from countries where the HBV prevalence rate is greater than $2 \%$. Screening will facilitate the provision of medical and public health services for infected patients and their families and provide public health data on the burden of disease in immigrant populations. The screening test should not be used to prohibit immigration.

From the University of Nebraska Medical Center, Omaha, Nebraska; Marshfield Clinic Research Foundation, Marshfield, Wisconsin; Yale University School of Medicine, New Haven, Connecticut; Brown University, Providence, Rhode Island; University of California, San Francisco, and San Francisco General Hospital, San Francisco, California; The University of Alabama School of Medicine, Birmingham, Alabama; University of Washington School of Medicine and Swedish PhysiciansCentral Seattle Clinic, Seattle, Washington; Mayo Clinic, Rochester, Minnesota; University of Virginia, Charlottesville, Virginia; University of Vermont College of Medicine, Burlington, Vermont; and American Melanoma Foundation, San Diego, California.

Potential Financial Conflicts of Interest: None disclosed.

Requests for Single Reprints: Reprints are available from the NIH Consensus Development Program Web site (www.consensus.nih.gov) and in print through the NIH Consensus Development Program Information Center (888-644-2667).

Current author addresses are available at www.annals.org.

\section{Reference}

1. Shamliyan TA, MacDonald R, Shaukat A, Taylor BC, Yuan JM, Johnson JR, et al. Antiviral therapy for adults with chronic hepatitis B: a systematic review for a National Institutes of Health Consensus Development Conference. Ann Intern Med. 2009;150:111-24. 
Current Author Addresses: Dr. Sorrell: Section of Gastroenterology and Hepatology, University of Nebraska Medical Center, $983285 \mathrm{Ne}$ braska Medical Center, Omaha, NE 68198-3285.

Dr. Belongia: Marshfield Clinic Research Foundation, 1000 North Oak Avenue, ML2, Marshfield, WI 54449.

Dr. Costa: Department of Pathology, Yale University School of Medicine, 310 Cedar Street, BML 348, New Haven, CT 06510.

Dr. Gareen: Department of Community Health, Center for Statistical Sciences, Brown University, Box G-S121-7, 121 South Main Street, Providence, RI 02912.

Dr. Grem: Department of Internal Medicine, Section of Oncology and Hematology, University of Nebraska Medical Center, 987680 Nebraska Medical Center, Omaha, NE 68198-7680.

Dr. Inadomi: San Francisco General Hospital, 1001 Potrero Avenue, Room NH 3D5, San Francisco, CA 94110.

Dr. Kern: The University of Alabama School of Medicine, 1600 6th Avenue South, CHB 128, Birmingham, AL 35216.

Dr. McHugh: Swedish Physicians-Central Seattle Clinic, 1600 East Jefferson, Suite 510, Seattle, WA 98122.

Dr. Petersen: College of Medicine, Mayo Clinic, 200 First Street Southwest, Rochester, MN 55905.

Dr. Rein: Division of Infectious Diseases and International Health, University of Virginia, 109 Sturbridge Road, Charlottesville, VA 22091.

Dr. Strader: Division of Gastroenterology/Hepatology, Fletcher Allen Health Care, University of Vermont College of Medicine, 111 Colchester Avenue, Smith 247A, Burlington, VT 05401.

Mr. Trotter: American Melanoma Foundation, 17459 Drayton Hall Way, San Diego, CA 92128-2043.

\section{APPENDIX}

\section{Consensus Development Panel}

Michael F. Sorrell, MD (Panel and Conference Chairperson), Section of Gastroenterology and Hepatology, University of $\mathrm{Ne}$ braska Medical Center, Omaha, Nebraska; Edward A. Belongia, MD, Epidemiology Research Center, Marshfield Clinic Research Foundation, Marshfield, Wisconsin; Jose Costa, MD, Department of Pathology, Yale University School of Medicine, New Haven, Connecticut; Ilana F. Gareen, PhD, Department of Community Health, Center for Statistical Sciences, Brown University, Providence, Rhode Island; Jean L. Grem, MD, Department of Internal Medicine, Section of Oncology and Hematology, University of Nebraska Medical Center, Omaha, Nebraska; John M. Inadomi, MD, Policy and Economics (HOPE) Research Program, University of California, San Francisco, and Clinical Gastroenterology, San Francisco General Hospital, San Francisco, California; Earl R. Kern, PhD, Department of Pediatrics, The University of Alabama School of Medicine, Birmingham, Alabama; James A. McHugh, MD, Department of Family Medicine, University of Washington School of Medicine, and Family Medicine, Swedish Medical Center, Swedish Physicians-Central Seattle Clinic, Seattle, Washington; Gloria M. Petersen, PhD, College of Medicine, Mayo Clinic, Rochester, Minnesota; Michael F. Rein, MD, Division of Infectious Diseases and International Health, University of Virginia, Charlottesville, Virginia; Doris B. Strader, MD, Division of Gastroenterology/Hepatology, Fletcher Allen Health Care, University of Vermont College of Medicine, Burlington, Vermont; $\mathrm{H}$. Thomas Trotter, MS, U.S. Navy (Ret.), American Melanoma Foundation, San Diego, California.

\section{Speakers}

Chien-Jen Chen, ScD, MPH, Genomics Research Center, Academia Sinica, and National Taiwan University, Nankang, Taipei City, Taiwan; Raymond T. Chung, MD, Harvard Medical School and Liver Transplant Program, Massachusetts General Hospital, Boston, Massachusetts; Adrian M. Di Bisceglie, MD, Division of Gastroenterology and Hepatology, St. Louis University School of Medicine, St. Louis, Missouri; Jules L. Dienstag, MD, Harvard Medical School, Boston, Massachusetts; Robert J. Fontana, MD, Division of Gastroenterology, Department of Internal Medicine, University of Michigan Medical School, Ann Arbor, Michigan; Marc G. Ghany, MD, Liver Diseases Branch, National Institute of Diabetes and Digestive and Kidney Diseases, National Institutes of Health, Bethesda, Maryland; Jenny Heathcote, MD, Division of Patient-Based Clinical Research, Gastroenterology, Toronto Western Hospital, University of Toronto, Toronto, Ontario, Canada; Jay H. Hoofnagle, MD, Liver Disease Research Branch, Division of Digestive Diseases and Nutrition, National Institute of Diabetes and Digestive and Kidney Diseases, National Institutes of Health, Bethesda, Maryland; W. Ray Kim, MD, MSc, MBA, Division of Gastroenterology and Hepatology, Department of Internal Medicine, Mayo Clinic, Rochester, Minnesota; David E. Kleiner, MD, PhD, Postmortem Section, Laboratory of Pathology, National Cancer Institute, National Institutes of Health, Bethesda, Maryland; T. Jake Liang, MD, Liver Diseases Branch, National Institute of Diabetes and Digestive and Kidney Diseases, National Institutes of Health, Bethesda, Maryland; Anna S.F. Lok, MD, Division of Gastroenterology, University of Michigan Health System, Ann Arbor, Michigan; Brian J. McMahon, MD, Liver Disease and Hepatitis Program, Alaska Native Medical Center, and Arctic Investigations Program, Centers for Disease Control and Prevention, Anchorage, Alaska; Robert P. Perrillo, MD, Hepatology Division and Liver Fellowship, Baylor University Medical Center, Dallas, Texas; Marion G. Peters, MD, MBBS, Hepatology Research, University of California, San Francisco, San Francisco, California; Eugene R. Schiff, MD, Schiff Liver Institute and Center for Liver Diseases, University of Miami School of Medicine, Miami, Florida; Aasma Shaukat, MD, MPH, University of Minnesota, Minneapolis, Minnesota; Brent C. Taylor, PhD, MPH, Center for Chronic Disease Outcomes Research, Minneapolis Veterans Affairs Medical Center, and University of Minnesota, Minneapolis, Minnesota; Norah A. Terrault, MD, MPH, Division of Gastroenterology, Department of Medicine, University of California, San Francisco, San Francisco, California; Chloe L. Thio, MD, Division of Infectious Diseases, Johns Hopkins School of Medicine, Baltimore, Maryland; Cindy M. Weinbaum, MD, MPH, Prevention Branch Research and Evaluation Team, Division of Viral Hepatitis, Centers for Disease Control and Prevention, Atlanta, Georgia; Timothy J. Wilt, MD, MPH, Center for Chronic Disease Outcomes Research, Minneapolis Veterans Affairs Medical Center and Minnesota Agency for Healthcare Research and Quality Evidence-Based Practice Center, University of Minnesota, Minneapolis, Minnesota. 


\section{Planning Committee}

Jay H. Hoofnagle, MD, Liver Disease Research Branch, Division of Digestive Diseases and Nutrition, National Institute of Diabetes and Digestive, and Kidney Diseases, National Institutes of Health, Bethesda, Maryland; Lisa Ahramjian, MS, Office of Medical Applications of Research, Office of the Director, National Institutes of Health, Bethesda, Maryland; Shilpa Amin, MD, MBSc, Evidence-based Practice Centers Program, Center for Outcomes and Evidence, Agency for Healthcare Research and Quality, Rockville, Maryland; David Atkins, MD, MPH, Center for Outcomes and Evidence, Agency for Healthcare Research and Quality, Rockville, Maryland; Diana Berard, Enteric and Hepatic Diseases Branch, Division of Microbiology and Infectious Diseases, National Institute of Allergy and Infectious Diseases, National Institutes of Health, Bethesda, Maryland; Robin Biswas, MD, Division of Emerging and Transfusion-Transmitted Diseases, U.S. Food and Drug Administration, Rockville, Maryland; Timothy Block, PhD, Drexel Institute for Biotechnology and Virology Research, Microbiology and Immunology, Drexel University College of Medicine, Doylestown, Pennsylvania; John S. Cole III, PhD, Cancer Etiology Branch, Division of Cancer Biology, National Cancer Institute, National Institutes of Health, Bethesda, Maryland; Jennifer Miller Croswell, MD, Office of Medical Applications of Research, Office of the Director, National Institutes of Health, Bethesda, Maryland; Jules L. Dienstag, MD, Harvard Medical School, Boston, Massachusetts; Edward Doo, MD, Liver Disease Research Programs, National Institute of Diabetes and Digestive and Kidney Diseases, National Institutes of Health, Bethesda, Maryland; James Everhart, MD, MPH, Epidemiology and Clinical Trials Branch, Division of Digestive Diseases and Nutrition, National Institute of Diabetes and Digestive and Kidney Diseases, National Institutes of Health, Bethesda, Maryland; Russell Fleischer, PA-C, MPH, Division of Antiviral Products, U.S. Food and Drug Administration, Silver Spring, Maryland; Barnett S. Kramer, MD, MPH, Office of Medical Applications of Research, Office of the Direc- tor, National Institutes of Health, Bethesda, Maryland; Anna S.F. Lok, MD, Division of Gastroenterology, University of Michigan Health System, Ann Arbor, Michigan; Willis C. Maddrey, MD, University of Texas Southwestern Medical Center at Dallas, Dallas, Texas; Brian J. McMahon, MD, Liver Disease and Hepatitis Program, Alaska Native Medical Center, and Arctic Investigations Program, Centers for Disease Control and Prevention, Anchorage, Alaska; Robert P. Perrillo, MD, Hepatology Division, Liver Fellowship, Baylor University Medical Center, Dallas, Texas; Michael F. Sorrell, MD (Panel and Conference Chairperson), Section of Gastroenterology and Hepatology, University of Nebraska Medical Center, Omaha, Nebraska; David L. Thomas, MD, Infectious Diseases Viral Hepatitis Center, The Johns Hopkins University School of Medicine, Baltimore, Maryland; Cindy Weinbaum, MD, MPH, Prevention Branch Research and Evaluation Team, Division of Viral Hepatitis, Centers for Disease Control and Prevention, Atlanta, Georgia; Ian T. Williams, PhD, MS, Epidemiologic Research and Field Investigations Team, Division of Viral Hepatitis, Centers for Disease Control and Prevention, Atlanta, Georgia.

\section{Conference Sponsors}

National Institute of Diabetes and Digestive and Kidney Diseases (Griffin P. Rodgers, MD, Director), Office of Medical Applications of Research (Barnett S. Kramer, MD, MPH, Director), and The Johns Hopkins University School of Medicine, Educational Provider (Todd Dorman, MD, FCCM, Associate Dean and Director, Continuing Medical Education).

\section{Conference Cosponsors}

National Cancer Institute (John E. Niederhuber, MD, Director) and National Institute of Allergy and Infectious Diseases (Anthony S. Fauci, MD, Director).

\section{Conference Partners}

Centers for Disease Control and Prevention (Julie Louise Gerberding, MD, MPH, Director) and U.S. Food and Drug Administration (Andrew C. von Eschenbach, MD, Commissioner). 